\title{
COMPOSIÇÃO MINERAL, SANIDADE E QUALIDADE DE MAÇÃS EM POMARES CONVENCIONAIS E ORGÂNICOS ${ }^{1}$
}

\author{
JOÃO JOSÉ STÜPP², ELIETE DE FÁTIMA FERREIRA DA ROSA, \\ CASSANDRO VIDAL TALAMINI DO AMARANTE ${ }^{4}$, ÁLVARO LUIZ MAFRA ${ }^{5}$, \\ MARI LUCIA CAMPOS
}

RESUMO - O objetivo deste trabalho foi avaliar os efeitos dos sistemas de manejo convencional e orgânico sobre a composição mineral, sanidade e qualidade de maçãs das cultivares Royal Gala e Fuji. O estudo foi realizado em São Joaquim-SC, nas safras de 2008/2009 e 2009/2010, em um Cambissolo Húmico. O manejo orgânico, em ambas as cultivares, aumentou a área foliar média e específica e os teores foliares de $\mathrm{K} \mathrm{e} \mathrm{Cu}$, e em 'Royal Gala' aumentou o Ca foliar. Na maturação comercial, frutos orgânicos apresentaram maiores valores de percentual de cor vermelha na epiderme, firmeza de polpa, danos por mosca-das-frutas, teores de $\mathrm{Cu}$ e relações $\mathrm{K} / \mathrm{Ca}, \mathrm{Mg} / \mathrm{Ca}$ e $(\mathrm{K}+\mathrm{Mg}) / \mathrm{Ca}$ na polpa, em ambas as cultivares, e de teor de $\mathrm{K}$ na polpa em 'Royal Gala'. As plantas no sistema orgânico apresentaram valores inferiores de teores foliares de clorofila e $\mathrm{N}$, rendimento, incidência de frutos com sarna da macieira, bem como frutos com menores teores de sólidos solúveis e de Ca na polpa, em ambas as cultivares, menores danos por queimadura de sol em 'Royal Gala' e menor teor de $\mathrm{K}$ na casca dos frutos em 'Fuji'. A produção orgânica de maçãs pode ser viável, porém necessita da adoção de tecnologia eficaz para o controle de danos por mosca-das-frutas.

Termos para indexação: Malus domestica, composição mineral de maçãs, cobre em maçã orgânica, "russeting", mosca-das-frutas.

\section{MINERAL COMPOSITION, PHYTOSSANITY AND FRUIT QUALITY OF CONVENTIONAL AND ORGANIC APPLE ORCHARDS}

\begin{abstract}
The objective of this study was to assess the effects of conventional and organic management systems on mineral composition, incidence of pests and fruit quality attributes of 'Royal Gala' and 'Fuji apples. This study was carried out in São Joaquim-SC, in 2008/09 and 2009/10, on a Humic Inceptisol. Apple trees under organic management system had leaves with higher mean and specific area, and higher $\mathrm{K}$ and $\mathrm{Cu}$ contents in both cultivars, and higher Ca content in 'Royal Gala'. Organic apples at commercial maturity had higher values of percentage of blush in apples skin, flesh firmness, fruit fly damage, higher K/ $\mathrm{Ca}, \mathrm{Mg} / \mathrm{Ca}$ and $(\mathrm{K}+\mathrm{Mg}) / \mathrm{Ca}$ ratios in flesh fruit, and higher $\mathrm{Cu}$ content in both cultivars, as well as higher $\mathrm{K}$ content in the flesh of 'Royal Gala' apples. Apples trees of organic system had lower values of chlorophyll and $\mathrm{N}$ contents in the leaves, lower yield and lower incidence of fruits with apple scab, fruits with lower soluble solids and $\mathrm{Ca}$ contents in the flesh fruit, in both cultivars, as well as lower incidence of sunburn in fruits of 'Royal Gala' and lower fruit skin K content in 'Fuji' apples. Organic apple production system is viable, since efficient technologies are adopted to prevent the fruit fly damage.
\end{abstract}

Index terms: Malus domestica, apples mineral composition, copper in organic apples, russeting, fruit fly.

\footnotetext{
'(Trabalho 398-13). Recebido em : 15-10-2013. Aceito para publicação em: 27-10-2014.

${ }^{2}$ Dr., Professor do Instituto Federal Catarinense, Câmpus Rio do Sul, Estrada do Redentor, 5665, CEP 89160-000 Rio do Sul-SC. E-mail: jjstupp@gmail.com

${ }^{3}$ M.Sc., Doutoranda em Manejo do Solo, (CAV), Universidade do Estado de Santa Catarina (UDESC). Av. Luiz de Camões, 2090, CEP 88520-000. Lages-SC, Brasil. E-mail: elietedarosa@hotmail.com

${ }^{4}$ Ph.D, Bolsista de Produtividade em Pesquisa do CNPq, Professor do Departamento de Agronomia, CAV/UDESC, Av. Luiz de Camões, 2090, CEP 88520-000, Lages-SC, Brasil. E-mail: amarante@cav.udesc.br. Autor para correspondência.

${ }^{5}$ Dr., Professor do Departamento de Solos e Recursos Naturais, CAV/UDESC, Lages-SC, Brasil. E-mail: alvaro.mafra@udesc.br, a2mlc@cav.udesc.br
} 


\section{INTRODUÇÃO}

A fruticultura de clima temperado destaca-se entre as atividades agrícolas pelo desenvolvimento de práticas culturais intensivas. As alternativas para o manejo dos pomares são também condicionadas pelo sistema de manejo adotado, sendo no caso da fruticultura destacados os sistemas convencional e orgânico de produção.

O sistema convencional de produção apresenta função importante na produção de alimentos, mas é dependente do aporte de insumos químicos, como fertilizantes e pesticidas. Essas práticas agrícolas trazem preocupações a produtores e consumidores, os quais têm buscado alimentos saudáveis, livres de substâncias contaminantes e com bom aspecto visual. Neste sentido, o sistema orgânico de produção surge como uma alternativa, pois requer menos insumos externos à propriedade, exclui o uso de agrotóxicos e adubos solúveis e utiliza técnicas alternativas de aporte de nutrientes, como a permanência da cobertura vegetal e a aplicação de adubos orgânicos (HOLB et al., 2012). Porém, o simples fato de ser orgânico não garante a sustentabilidade do sistema (AMARANTE et al., 2008). Para ser sustentável, o sistema deve ser administrado de forma socialmente responsável, preservar os recursos naturais, proteger o meio ambiente, ter produtividade satisfatória e ser lucrativo (REGANOLD et al., 2001; AMARANTE et al., 2008).

Os sistemas orgânicos de produção são considerados menos eficientes, apresentando maiores riscos de doenças e rendimento inferior em relação aos sistemas convencional e integrado (HOLB et al., 2012). Segundo Peck et al. (2011), rendimentos menores em pomares orgânicos de maçãs são, provavelmente, resultado da insatisfatória gestão da produção e da maior pressão de pragas, doenças e deficiências nutricionais.

Alguns trabalhos vêm sendo realizados visando a comparar atributos químicos e físicos do solo (PECK et al., 2011), atributos microbiológicos do solo (MALUCHE-BARETTA et al., 2006) e rendimento e qualidade de frutos produzidos nos sistemas convencional e orgânico de produção (ROUSSOS;GASPARATOS, 2009; HOLB et al., 2012). Alguns autores não observaram substanciais diferenças entre maçãs de pomares orgânicos e integrados em termos de qualidade de frutos na colheita (JÖNSSON, 2007). Outros autores têm citado melhor qualidade de maçãs orgânicas em relação às obtidas de pomares integrados ou convencionais (WEIBEL et al., 2004; PECK et al.,
2006). Outros ainda observaram mais semelhanças que diferenças entre os sistemas de manejo quanto à qualidade dos frutos (ROUSSOS; GASPARATOS, 2009). Por isso, são necessárias mais pesquisas científicas para a elucidação destas e outras questões, avaliando e comparando os dois sistemas de produção de maçãs.

Diante disso, o presente estudo teve como objetivo avaliar o efeito dos sistemas de manejo convencional e orgânico, sobre a composição mineral, sanidade, rendimento e qualidade de frutos, em pomares de maçãs 'Royal Gala' e 'Fuji', nas condições da região Sul do Brasil.

\section{MATERIAL E MÉTODOS}

O presente estudo foi realizado nas safras 2008/2009 e 2009/2010, no município de São Joaquim- SC ( $\left.28^{\circ} 12^{\prime} 15^{\prime \prime} \mathrm{S}, 50^{\circ} 03^{\prime} 48^{\prime \prime} \mathrm{O}\right)$, a uma altitude média de $1.350 \mathrm{~m}$. O clima é mesotérmico úmido $(\mathrm{Cfb})$, segundo a classificação de Köppen, com verão brando. As chuvas são bem distribuídas durante todo o ano, com menor precipitação de dezembro a fevereiro.

As áreas estudadas constituíram-se de um pomar de maçãs conduzido sob o sistema convencional (PC) e um conduzido sob o sistema orgânico de produção (PO), com filas alternadas da cultivar 'Royal Gala' e 'Fuji' sobre o porta-enxerto M-9, ambos implantados em 2001. Os pomares distam entre si, aproximadamente, $1 \mathrm{~km}$, o que reduz possíveis diferenças de solo e microclima.

O solo dos pomares foi classificado como Cambissolo Húmico. Antes da implantação do experimento, foram avaliadas características como tipo e textura do solo, a fim de verificar possíveis diferenças no solo entre os pomares que pudessem influenciar os resultados. As características químicas do solo encontram-se na Tabela 1.

O pomar convencional recebeu, na fase de implantação, $40 \mathrm{Mg} \mathrm{ha}^{-1}$ de calcário ( $70 \%$ dolomítico e $30 \%$ calcítico), incorporado na camada de $0-40 \mathrm{~cm}$, $300 \mathrm{~g} \mathrm{~m}^{-2}$ de superfosfato triplo, aplicado na faixa de plantio das macieiras, e $30 \mathrm{~kg} \mathrm{ha}^{-1}$ de bórax. $\mathrm{Na}$ formação do pomar, foram feitas três aplicações anuais de ureia $\left(30,45\right.$ e $60 \mathrm{~g}^{\text {planta }}{ }^{-1}$, no primeiro, segundo e terceiro anos, respectivamente). A adubação de reposição anual consistiu em $50 \mathrm{~g}$ de ureia planta ${ }^{-1}$, na fase de brotação, e $100 \mathrm{~g}$ de cloreto de potássio planta ${ }^{-1}$, no mês de dezembro. Após a colheita, foi aplicada cal hidratada, proveniente das câmaras de frigoconservação e, na floração, cálcio quelatizado $(0,7 \%)$ e ácido bórico $(0,5 \%)$. De dezembro até a colheita, foram feitas pulverizações 
foliares quinzenais com cloreto de cálcio $(0,6 \%)$. A quebra de dormência foi feita através de pulverização combinada de cianamida hidrogenada $(0,25 \%)$ e óleo mineral (4\%), e o raleio de frutos, após o estabelecimento dos mesmos, com raleantes químicos, complementado manualmente, deixandose até dois frutos por cacho floral. O monitoramento da mosca-das-frutas foi feito com armadilhas caçamosca; e o controle, com inseticidas específicos (Fenitrotiona, Metidationa e Malathion), quando o nível de controle era atingido. O controle da sarnada- macieira [Venturia inaequalis (Cooke)] foi feito com fungicidas específicos, recomendados para o manejo convencional (Difenoconazol, Pirimethanil, Miclobutanil, Captan, Trifloxistrobina e CresoximMetílico), sendo que nenhum dos produtos utilizados foi à base de cobre. A aplicação de fungicidas foi feita a partir de informações monitoradas pelas Estações de Avisos Fitossanitários (temperatura, umidade relativa do ar e o período de molhamento foliar), informando as condições favoráveis à liberação de ascosporos e de infecção. No período crítico para a ocorrência da sarna (que se inicia com a brotação da macieira e prolonga-se até o final de novembro, quando cessa a liberação de ascosporos), a aplicação de fungicidas foi feita em intervalos de sete a dez dias. O manejo da cobertura verde foi feito com duas roçadas por estação de crescimento.

No pomar orgânico, o solo foi corrigido no plantio com $10 \mathrm{Mg} \mathrm{ha}^{-1}$ de calcário (30\% dolomítico e $70 \%$ calcítico), $350 \mathrm{~kg} \mathrm{ha}^{-1}$ de fosfatos $(60 \%$ solúvel e $40 \%$ natural), $30 \mathrm{~kg} \mathrm{ha}^{-1}$ de bórax e $2 \mathrm{~kg} \mathrm{planta}^{-1} \mathrm{de}$ cama de aves. A adubação de crescimento consistiu na aplicação anual de $1 \mathrm{~kg}$ planta $^{-1}$ de esterco de peru e na introdução de espécies leguminosas, como adubação verde. A reposição anual de nutrientes foi feita com $2 \mathrm{~kg}$ planta $^{-1}$ de esterco de aves e através da adubação verde, com espécies cultivadas e espontâneas. A quebra de dormência foi natural, pelo somatório das horas de frio ocorridas no período. $\mathrm{O}$ raleio foi manual, sendo deixados até dois frutos por cacho floral. O controle da sarna-da-macieira deuse através de aplicações com calda sulfocálcica (24 aplicações/safra) e produtos à base de cobre (duas aplicações de bordasul e uma de calda bordalesa a 1\%, em 2008, e apenas uma aplicação em 2010 de calda bordalesa a $1 \%$, em pós-colheita). O manejo da cobertura verde foi feito com duas roçadas por estação de crescimento.

Em cada pomar, foram selecionadas aleatoriamente dez plantas de cada cultivar, sendo que cada uma constituía-se numa unidade experimental. Nestas plantas, realizaram-se avaliações quanto à composição mineral, floração, frutificação, produção e qualidade de frutos.

O desenvolvimento reprodutivo das macieiras foi avaliado pela contagem do número de cachos florais em dois ramos laterais/planta previamente selecionados, sendo expresso quanto ao número de cachos florais por $\mathrm{cm}^{2}$ de secção transversal de ramo. A frutificação efetiva foi avaliada nestes mesmos ramos, pela contagem do número total de frutos com diâmetro $\geq 1,0 \mathrm{~cm}, 30$ dias após a plena floração, antes do raleio de frutos, sendo expressa quanto ao número de frutos por $\mathrm{cm}^{2}$ de secção transversal de ramo e número de frutos por cacho floral.

Para a análise mineral do tecido foliar, procedeu-se à coleta de 100 folhas, no terço médio de lançamentos do ano, em cada planta selecionada, cerca de trinta dias antes da colheita dos frutos, para a avaliação dos teores de de $\mathrm{Ca}, \mathrm{Mg}, \mathrm{K} \mathrm{e} \mathrm{N}$, segundo metodologia descrita por Tedesco et al. (1995). Os teores de $\mathrm{Cu}$ no tecido foliar foram determinados por digestão nitroperclórica, conforme descrito por Roussos e Gasparatos (2009), e quantificados em espectrofotômetro de absorção atômica.

Realizaram-se também amostragens de 20 folhas por unidade experimental, coletadas no terço médio de lançamentos do ano, sendo as mesmas avaliadas em termos de teor total de clorofila, através de leituras com o clorofilômetro SPAD-502), área foliar (com um integrador de área foliar LI-COR modelo LI-3050A, em $\mathrm{cm}^{2}$ ), massa seca foliar (g) e área foliar específica $\left(\mathrm{cm}^{2} \mathrm{~g}^{-1}\right)$.

Na maturação comercial, todos os frutos dos dois ramos marcados de cada planta selecionada foram colhidos e avaliados quanto ao rendimento (número e massa dos frutos por $\mathrm{cm}^{2}$ de secção transversal do ramo, e massa média dos frutos). Para a determinação dos teores de $\mathrm{Ca}, \mathrm{Mg}, \mathrm{K}$ e $\mathrm{N}$ dos frutos, utilizaram-se 20 frutos por amostra, sendo avaliados separadamente os teores desses minerais na casca e na polpa dos frutos. Os frutos foram lavados com água destilada, realizando-se a remoção de toda a casca da superfície, numa espessura média de 0,2 $\mathrm{cm}$. A polpa e a casca dos frutos foram digeridas separadamente em uma mistura de ácido sulfúrico e peróxido de hidrogênio, como descrito por Amarante et al. (2008). O N foi quantificado por meio de destilação a vapor, utilizando equipamento semimicro Kjeldahl. $\mathrm{O} \mathrm{Ca}$ e o $\mathrm{Mg}$ foram determinados em espectrofotômetro de absorção atômica, e o K, em fotômetro de chama.

Amostras de 20 frutos por planta foram avaliadas quanto à firmeza de polpa $(\mathrm{N}$; com penetrômetro munido de ponteira com $11,1 \mathrm{~mm}$ de diâmetro), teor de sólidos solúveis (SS; ${ }^{\circ}$ Brix), índice 
de iodo-amido (em escala de 1 a 5, onde 1 indica maior e 5 menor conteúdo de amido), acidez titulável (AT; \% de ácido málico), percentual de cor vermelha da epiderme, severidade de "russeting" $\left(\mathrm{cm}^{2}\right.$ por fruto), número de sementes por fruto, e incidência (\%) de queimadura do sol, sarna-da-macieira e danos por mosca-das-frutas, segundo metodologia descrita por Amarante et al. (2008).

Os resultados obtidos foram submetidos à análise de variância $(p<0,05)$, utilizando-se do programa estatístico SAS, versão 9.0 (SAS Institute, 2002).

\section{RESULTADOS E DISCUSSÃO}

$\mathrm{O} \mathrm{pH}$ do solo estava neutro ou próximo da neutralidade em ambos os sistemas de manejo, o que indica adequada correção da acidez na fase de implantação (Tabela 1).

Macieiras 'Royal Gala' sob cultivo orgânico tiveram maiores teores de $\mathrm{Ca}$ e $\mathrm{K}$, e menores teores de $\mathrm{N}$ nas folhas, enquanto macieiras 'Fuji' sob manejo orgânico tiveram maiores teores de $\mathrm{K}$ e menores teores de $\mathrm{Ca}$ nas folhas, em relação a macieiras do sistema convencional (Tabela 2).

Sob cultivo orgânico, o teor de Ca na polpa dos frutos também foi menor, em ambas as cultivares (Tabela 2), possivelmente devido aos teores menores de Ca no solo dos pomares neste sistema de manejo (Tabela 1). Maçãs 'Fuji' do pomar orgânico tiveram menores teores de Ca na polpa e de $\mathrm{K}$ na casca, devido ao menor tamanho de células e de espaços intercelulares, os quais resultam em frutos com menor massa média nessa cultivar (Tabela 2).

Diferenças quanto aos teores de $\mathrm{Ca}$ nas folhas e frutos de 'Royal Gala' não foram observadas em estudo realizado por Amarante et al. (2008); contudo, para a cultivar Fuji, os autores verificaram teores maiores de $\mathrm{Ca}$ nas folhas, em plantas de pomares convencionais. Devido à restrita mobilidade do Ca na planta, não se observou relação entre os teores de Ca nas folhas e frutos (casca e polpa) de 'Royal Gala'. Frutos de 'Royal Gala' provenientes dos pomares convencional e orgânico, e de 'Fuji' do pomar convencional, apresentaram teores de $\mathrm{Ca}>40 \mathrm{mg} \mathrm{kg}^{-1}$ de massa fresca (Tabela 2), o que indica adequado suprimento de $\mathrm{Ca}$ nos frutos (AMARANTE et al., 2012). Por outro lado, maçãs 'Fuji' provenientes do pomar sob sistema orgânico tiveram níveis de Ca na polpa $<40 \mathrm{mg} \mathrm{kg}^{-1}$ de massa fresca. Além disso, teores menores de Ca nos frutos induzem ao aumento das relações $\mathrm{N} / \mathrm{Ca}, \mathrm{K} / \mathrm{Ca}$ e $(\mathrm{K}+\mathrm{Mg}) / \mathrm{Ca}$, o que, segundo Amarante et al. (2006a e 2006b), favorece a ocorrência de diversos distúrbios relacionados à deficiência de $\mathrm{Ca}$, comprometendo a qualidade das maçãs, principalmente durante o armazenamento.

A relação N/Ca em polpa de maçãs 'Fuji' foi significativamente maior nos frutos orgânicos, em relação aos do sistema convencional, devido aos menores teores de $\mathrm{Ca}$ na polpa dos frutos orgânicos (Tabela 2). Estes resultados corroboram os estudos realizados por Roussos e Gaspartos (2009), os quais verificaram maior relação $\mathrm{N} / \mathrm{Ca}$ em frutos produzidos no sistema orgânico. Todavia, Peck et al. (2006) relataram menor relação N/Ca em maçãs orgânicas em relação às provenientes de pomares sob manejo convencional. Segundo Amarante et al. (2012), maçãs com teor de $\mathrm{N}$ inferior a $500 \mathrm{mg} \mathrm{kg}^{-1}$ de massa fresca e valores da relação $\mathrm{N} / \mathrm{Ca}$ menores do que 14 apresentam menores riscos de ocorrência de "bitter pit". Teores excessivos de $\mathrm{N}$ aumentam o tamanho dos frutos, diluindo o $\mathrm{Ca}$ e aumentando a suscetibilidade dos mesmos para a expressão de distúrbios ligados à deficiência de $\mathrm{Ca}$ e a doenças, além de redução da cor vermelha da epiderme de maçãs (AMARANTE et al., 2012). Além disso, a concentração de $\mathrm{N}$, bem como a relação $\mathrm{N} / \mathrm{Ca}$ estão positivamente ligadas à produção de etileno, o qual induz a incidência de pingo-de- mel, reduzindo a adesão das células, o que resulta na perda de firmeza dos frutos (HOLB et al., 2012).

$\mathrm{O} \mathrm{K}$ tem relação direta com a qualidade de frutos, e seu excesso deve ser evitado em vista do risco de indução de deficiências de $\mathrm{Ca}$ e $\mathrm{Mg}$, e de aumento na incidência de "bitter pit" nos frutos, principalmente devido aos maiores valores das relações $\mathrm{K} / \mathrm{Ca}$ e $(\mathrm{K}+\mathrm{Mg}) / \mathrm{Ca}$. Frutos de 'Royal Gala', sob manejo convencional apresentaram relação $\mathrm{K} / \mathrm{Ca}$ na polpa $<25$ (Tabela 2), o que denota boa qualidade para preservação da firmeza de polpa. Dilmaghani et al. (2004) observaram correlação negativa entre a relação $\mathrm{K} / \mathrm{Ca}$ e a firmeza de polpa em maçãs. Esses autores citam a relação $\mathrm{K} / \mathrm{Ca} \leq 25$ como adequada para melhor preservação da firmeza de polpa, em pós-colheita. Por outro lado, maçãs 'Fuji', nos dois sistemas de manejo, e 'Royal Gala' sob sistema orgânico, tiveram relação $\mathrm{K} / \mathrm{Ca}>25$, evidenciando maior probabilidade de ocorrência de distúrbios fisiológicos relacionados à deficiência de $\mathrm{Ca}$, em pós-colheita.

Não foram observadas diferenças entre os sistemas de manejo quanto à relação $(\mathrm{K}+\mathrm{Mg}) / \mathrm{Ca}$ na casca de maçãs 'Royal Gala' e 'Fuji' (Tabela 2). Todavia, a relação $(\mathrm{K}+\mathrm{Mg}) / \mathrm{Ca}$ foi maior na polpa dos frutos dos pomares orgânicos em ambas as cultivares, devido aos teores maiores de $\mathrm{K}$ e menores de $\mathrm{Ca}$ na polpa de 'Royal Gala', e aos menores teores de Ca na 
polpa de 'Fuji', neste sistema de produção. De acordo com Amarante et al. (2008), a relação (K+Mg)/Ca no fruto é o atributo mais indicado para avaliar riscos de ocorrência de "bitter pit" em maçãs.

Com relação aos teores de $\mathrm{Mg}$ no tecido foliar de 'Royal Gala' sob manejo orgânico, verifica-se que os valores ficaram abaixo dos níveis adequados para a cultura da macieira (AMARANTE et al., 2006a) (Tabela 2). Igualmente, os valores de $\mathrm{Mg}$ na polpa dos frutos foram $<40 \mathrm{mg} \mathrm{kg}^{-1}$, o que está relacionado à menor quantidade de calcário dolomítico aplicado no pomar orgânico, o que resultou em aporte deficiente desse elemento para os frutos. No entanto, não se detectaram diferenças entre os sistemas de manejo quanto aos teores de $\mathrm{Mg}$ no tecido foliar e nos frutos das duas cultivares estudadas. Amarante et al. (2008) observaram maior teor de $\mathrm{Mg}$ em frutos de pomares convencionais, em maçãs 'Royal Gala' e 'Fuji'. Todavia, em folhas de 'Royal Gala', esses autores encontraram maiores teores de Mg no tecido nas plantas sob sistema de manejo orgânico.

$\mathrm{O}$ teor de $\mathrm{Cu}$ em folhas e frutos (casca e polpa) foi maior no sistema orgânico em relação ao convencional, em ambas as cultivares (Tabela 2). No tecido foliar, foram encontrados teores de $\mathrm{Cu}$ acima dos considerados normais (5 a $30 \mathrm{mg} \mathrm{kg}^{-1} \mathrm{de}$ massa seca) (ROUSSOS;GASPARATOS, 2009). Os altos teores de Cu são decorrentes das aplicações de caldas cúpricas na safra de 2008/2009, a fim de evitar infecção de sarna da macieira nas fases posteriores de desenvolvimento dos frutos. Alguns autores relacionam o $\mathrm{Cu}$ como indutor de "russeting" em frutos produzidos organicamente (PALMER et al., 2003), o que não foi observado neste estudo (Tabela 3), possivelmente em decorrência do baixo número de aplicações desses fungicidas.

Em 'Royal Gala' (Tabela 3), frutos do sistema convencional, exibiram maior incidência de queimadura por sol. Neste caso, o sistema de condução das plantas empregado tem influência fundamental na estruturação da copa e proteção dos frutos com relação à ação direta dos raios solares. Nos pomares do sistema convencional foi aplicado um sistema de poda mais severo, realizando-se inclusive a poda verde, visando aumento da produção de órgãos de frutificação e a otimização da interceptação de luz para máxima eficiência fotossintética. Contudo, isto expõe os frutos a maior incidência e intensidade luminosa, o que pode aumentar a temperatura da superfície dos frutos causando danos e perdas por queimadura de sol.

O teor de clorofila foliar e a área foliar específica foram significativamente maiores no sistema convencional, em 'Royal Gala' e 'Fuji' (Tabela 3), o que pode estar relacionado ao emprego da calda sulfocálcica, usada no controle da sarna-damacieira, nos pomares sob manejo orgânico, pois foram realizadas 24 pulverizações em cada ciclo cultural. Amarante et al. (2008) também observaram redução do teor de clorofila e da área foliar específica, em pomares orgânicos de 'Royal Gala' e 'Fuji', possivelmente decorrente do emprego de caldas à base de enxofre molhável (calda sulfocálcica) e de sulfato de cobre (calda bordalesa). O enxofre molhável, componente da calda sulfocálcica, é o possível responsável por danos em plantas, devido à redução na assimilação de $\mathrm{CO}_{2}$, reforçando esses efeitos tóxicos (PALMER et al., 2003).

O menor rendimento de frutos sob manejo orgânico está associado a danos provocados pela mosca-das-frutas (Anastrepha fraterculus, Wiedemann). Nos pomares sob manejo orgânico, 69,4 e $75 \%$ dos frutos de 'Royal Gala' e 'Fuji', respectivamente, foram danificados na safra de 2008/2009 (Tabela 3). A mosca-das-frutas é a principal praga dos frutos em fruteiras de clima temperado cultivadas no Sul do Brasil (GARCIA et al., 2003). Os danos causados por essa espécie são normalmente frequentes e severos. $\mathrm{O}$ ensacamento de frutos apresenta resultados satisfatórios na proteção dos mesmos contra os danos por mosca-das-frutas em pomares sob manejo orgânico (TEIXEIRA et al., 2011), porém implica aumento significativo da mão de obra e dos custos de produção. Todavia, para o sistema de manejo orgânico, constitui-se ainda no único método eficaz disponível para o controle da mosca-das-frutas.

Frutos produzidos sob o sistema de manejo convencional apresentaram maior incidência de sarna (Venturia inaequalis), em ambas as cultivares (Tabela 3). O maior índice observado nesse sistema pode estar relacionado com as aplicações anuais de N (ureia) em cobertura, o que pode afetar negativamente a resistência das culturas com relação às doenças fúngicas (PECK et al., 2006). As sucessivas aplicações de calda sulfocálcica no sistema orgânico reduziram a incidência de sarna nesse sistema de manejo.

Frutos provenientes do sistema de manejo orgânico apresentaram maior percentual de cor vermelha na casca (Tabela 3). A aparência dos frutos é um fator de suma importância, que influencia nas preferências do consumidor. Maçãs com coloração da casca vermelho-intensa ou vermelho-rajada sobre fundo amarelo, tanto no mercado interno quanto no externo, são a preferência da maioria, já que essas características visuais são associadas a teores maiores 
de açúcares e frutos saudáveis (LAYNE et al., 2002).

De modo semelhante ao relatado por alguns autores (PECK et al., 2006; AMARANTE et al., 2008), frutos provenientes do sistema orgânico tiveram maior firmeza de polpa do que os frutos do sistema convencional, na colheita (Tabela 3 ), indicando menor avanço no processo de amadurecimento. Os teores de SS foram superiores em frutos do pomar convencional (Tabela 3). Os maiores teores de SS nos frutos deste tratamento estão relacionados ao maior conteúdo de pectinas solúveis, uma vez que esses frutos apresentaram a menor firmeza de polpa.

TABELA 1 - Atributos químicos dos solos de pomares de macieiras sob sistemas de manejo convencional e orgânico, nas profundidades de 0-10 cm e 10-20 cm. Médias de 10 repetições de cada pomar, das cultivares Royal Gala e Fuji.

\begin{tabular}{|c|c|c|c|c|c|c|c|c|c|}
\hline \multirow[t]{2}{*}{ Pomar } & \multirow{2}{*}{$\begin{array}{c}\mathrm{pH} \\
\text { água }\end{array}$} & \multirow{2}{*}{$\begin{array}{c}\mathrm{pH} \\
\mathrm{CaCl}_{2} \\
\end{array}$} & \multirow{2}{*}{$\begin{array}{c}\mathrm{N}^{(2)} \\
\left(\mathrm{g} \mathrm{kg}^{-1}\right) \\
\end{array}$} & \multirow{2}{*}{\multicolumn{2}{|c|}{$\frac{\mathrm{P} \quad \mathrm{Cu}}{\left(\mathrm{mg} \mathrm{kg}^{-1}\right)}$}} & K & & $\mathrm{Mg}$ & $\mathrm{Al}$ \\
\hline & & & & & & \multicolumn{4}{|c|}{$\left(\mathrm{cmol}_{\mathrm{c}} \mathrm{dm}^{-3}\right)^{(3)}$} \\
\hline & \multicolumn{9}{|c|}{$0-10 \mathrm{~cm}$} \\
\hline & \multicolumn{9}{|c|}{ 'Royal Gala' } \\
\hline Convencional & $7,2 *(1)$ & $6,7^{*}$ & $6,6 \mathrm{~ns}$ & $24,9 *$ & $8,3 * * *$ & $0,68 \mathrm{~ns}$ & $16,9 * * *$ & $5,3 \mathrm{~ns}$ & $0,03 \mathrm{~ns}$ \\
\hline \multirow[t]{2}{*}{ Orgânico } & 6,7 & 6,2 & 6,2 & 44,0 & 13,4 & 0,89 & 12,2 & 4,7 & 0,04 \\
\hline & \multicolumn{9}{|c|}{ 'Fuji' } \\
\hline Convencional & $6,8 \mathrm{~ns}$ & $6,1 \mathrm{~ns}$ & $6,0 \mathrm{~ns}$ & $32,0^{*}$ & $11,0 * * *$ & $0,71 \mathrm{~ns}$ & $13,3 * * *$ & $4,2^{*}$ & $0,06 \mathrm{~ns}$ \\
\hline \multirow[t]{3}{*}{ Orgânico } & 6,7 & 6,2 & 5,1 & 12,8 & 13,9 & 0,76 & 8,3 & 3,3 & 0,05 \\
\hline & \multicolumn{9}{|c|}{$10-20 \mathrm{~cm}$} \\
\hline & \multicolumn{9}{|c|}{ 'Royal Gala' } \\
\hline Convencional & $6,8 \mathrm{~ns}$ & $6,2^{*}$ & $4,7 \mathrm{~ns}$ & $18,2 \mathrm{~ns}$ & $8,9 * * *$ & $0,31 *$ & $13,0 * * *$ & $4,9^{*}$ & $0,02 * * *$ \\
\hline \multirow[t]{2}{*}{ Orgânico } & 6,4 & 5,7 & 4,7 & 17,6 & 13,8 & 0,58 & 7,5 & 3,7 & 0,06 \\
\hline & \multicolumn{9}{|c|}{ 'Fuji' } \\
\hline Convencional & $6,7^{*}$ & $5,8 \mathrm{~ns}$ & $4,9 \mathrm{~ns}$ & $20,9^{*}$ & $11,5 \mathrm{~ns}$ & $0,46 \mathrm{~ns}$ & $12,5^{* * *}$ & $4,4^{*}$ & $0,06 \mathrm{~ns}$ \\
\hline Orgânico & 6,2 & 5,7 & 4,5 & 6,2 & 12,6 & 0,38 & 6,8 & 3,5 & 0,05 \\
\hline
\end{tabular}

(1) Níveis de significância para diferenças entre pomares convencionais e orgânicos, para cada atributo, na mesma profundidade do solo (ns: não significativo; * e***: significativo aos níveis de probabilidade de 0,05 e 0,001 , respectivamente).

${ }^{(2)}$ Nitrogênio total.

(3) Valores trocáveis. 
TABELA 2 - Composição mineral de folhas ( $\mathrm{g} \mathrm{kg}^{-1}$ de massa seca) e frutos (casca e polpa, em mg kg-1 $\mathrm{de}^{-}$ massa fresca), e relações minerais na casca e polpa dos frutos, em macieiras 'Royal Gala' e 'Fuji', de pomares manejados sob os sistemas convencional e orgânico. Médias de 10 repetições de cada sistema de manejo, e das safras 2008/09 e 2009/10.

\begin{tabular}{|c|c|c|c|c|c|c|c|c|c|}
\hline Pomar & $\mathrm{Ca}$ & $\mathrm{K}$ & $\mathrm{Mg}$ & $\mathrm{N}$ & $\mathrm{Cu}$ & $\mathrm{K} / \mathrm{Ca}$ & $\mathrm{Mg} / \mathrm{Ca}$ & $\mathrm{N} / \mathrm{Ca}$ & $\mathrm{K}+\mathrm{Mg} / \mathrm{Ca}$ \\
\hline & \multicolumn{9}{|c|}{ 'Royal Gala' } \\
\hline & \multicolumn{9}{|c|}{ Folha } \\
\hline Convencional & $10,5^{*}$ & $20,8 * * *$ & $2,85 \mathrm{~ns}$ & $28,9 * *$ & $0,001 * * *$ & - & - & - & - \\
\hline Orgânico & 12,6 & 24,3 & 2,15 & 26,4 & 0,046 & - & - & - & - \\
\hline \multirow[t]{2}{*}{ CV $(\%)$} & 16,2 & 7,5 & 31,0 & 7,0 & 29,8 & - & - & - & - \\
\hline & \multicolumn{9}{|c|}{ Fruto - casca } \\
\hline Convencional & $153 \mathrm{~ns}$ & $1096 \mathrm{~ns}$ & $185 \mathrm{~ns}$ & $491 \mathrm{~ns}$ & $5,4 * * *$ & $7,5 \mathrm{~ns}$ & $1,26 \mathrm{~ns}$ & $3,3 \mathrm{~ns}$ & $8,7 \mathrm{~ns}$ \\
\hline Orgânico & 135 & 1050 & 196 & 451 & 9,5 & 7,8 & 1,49 & 3,4 & 9,3 \\
\hline \multirow[t]{2}{*}{ CV (\%) } & 22,0 & 21,7 & 15,3 & 12,2 & 11,2 & 27,8 & 25,7 & 23,8 & 26,4 \\
\hline & \multicolumn{9}{|c|}{ Fruto - polpa } \\
\hline Convencional & $58,9 * *$ & $1152 * * *$ & $37,8 \mathrm{~ns}$ & $259 \mathrm{~ns}$ & $2,9 * * *$ & $19,7 * * *$ & $0,64 *$ & $4,4 \mathrm{~ns}$ & $20,3 * * *$ \\
\hline Orgânico & 49,4 & 1495 & 39,7 & 258 & 5,7 & 30,7 & 0,82 & 5,2 & 31,5 \\
\hline \multirow[t]{3}{*}{$\mathrm{CV}(\%)$} & 13,3 & 13,8 & 13,4 & 17,4 & 14,5 & 16,9 & 22,4 & 21,2 & 16,9 \\
\hline & \multicolumn{9}{|c|}{ 'Fuji' } \\
\hline & \multicolumn{9}{|c|}{ Folha } \\
\hline Convencional & $14,8^{*}$ & $18,7 *$ & $2,75 \mathrm{~ns}$ & $29,9 \mathrm{~ns}$ & $0,003 * * *$ & - & - & - & - \\
\hline Orgânico & 13,2 & 20,2 & 3,04 & 29,6 & 0,053 & - & - & - & - \\
\hline \multirow[t]{2}{*}{$\mathrm{CV}(\%)$} & 9,9 & 6,7 & 19,0 & 6,0 & 45,7 & - & - & - & - \\
\hline & \multicolumn{9}{|c|}{ Fruto - casca } \\
\hline Convencional & $202 n s$ & $1832 * * *$ & $263 n s$ & $670 \mathrm{~ns}$ & $4,8 * * *$ & $10,0 \mathrm{~ns}$ & $1,44 \mathrm{~ns}$ & $3,6 \mathrm{~ns}$ & $11,5 \mathrm{~ns}$ \\
\hline Orgânico & 162 & 1525 & 233 & 693 & 9,1 & 10,1 & 1,54 & 4,5 & 11,6 \\
\hline \multirow[t]{2}{*}{$\mathrm{CV}(\%)$} & 35,4 & 9,2 & 14,6 & 10,1 & 19,0 & 31,7 & 31,1 & 29,3 & 31,2 \\
\hline & \multicolumn{9}{|c|}{ Fruto - polpa } \\
\hline Convencional & 51,9 & $1461 \mathrm{~ns}$ & $38,7 \mathrm{~ns}$ & $218 \mathrm{~ns}$ & $2,9 * * *$ & 28,6 & $0,76^{* *}$ & $4,3 *$ & $29,4 * * *$ \\
\hline Orgânico & 38,4 & 1496 & 36,7 & 205 & 5,5 & 39,1 & 0,96 & 5,4 & 40,1 \\
\hline $\mathrm{CV}(\%)$ & 13,7 & 10,4 & 7,2 & 16,4 & 12,1 & 14,2 & 15,1 & 22,9 & 13,9 \\
\hline
\end{tabular}

(*) Níveis de significância para diferenças entre pomares convencionais e orgânicos, para cada atributo mineral avaliado (ns: não significativo. ${ }^{* *}$ e ***: significativo aos níveis de probabilidade de 0,$05 ; 0,01$ e 0,001 , respectivamente). 
TABELA 3 - Atributos foliares, de floração, frutificação efetiva, rendimento e maturação e qualidade dos frutos, em macieiras 'Royal Gala' e 'Fuji', de pomares sob sistemas de manejo convencional e orgânico. Os valores representam a média de 10 repetições de cada pomar, e duas safras (2008/09 e 2009/10), exceto para os dados de mosca-das-frutas, que correspondem a uma safra $(2008 / 09)$.

\begin{tabular}{|c|c|c|c|c|}
\hline Atributos & $\begin{array}{c}\text { Pomar } \\
\text { Convencional }\end{array}$ & $\begin{array}{c}\text { Pomar } \\
\text { Orgânico }\end{array}$ & Significância ${ }^{(1)}$ & $\mathrm{CV}(\%)$ \\
\hline & \multicolumn{4}{|c|}{ 'Royal Gala' } \\
\hline Massa seca de 100 folhas (g) & 7,0 & 6,2 & $* *$ & 7,9 \\
\hline Teor de clorofila foliar (leitura SPAD) & 45,7 & 42,9 & $* * *$ & 3,6 \\
\hline No de cachos florais $\mathrm{PCSR}^{(2)}$ & 10,2 & 13,7 & ns & 35,0 \\
\hline $\mathrm{N}^{\circ}$ de frutos PCSR & 2,2 & 1,2 & $* * *$ & 30,6 \\
\hline $\mathrm{N}^{o}$ de frutos $\mathrm{PCF}^{(3)}$ & 0,8 & 0,36 & $* *$ & 53,6 \\
\hline Massa de frutos $(\mathrm{kg})$ PCSR & 0,25 & 0,18 & $*$ & 32,0 \\
\hline Massa média de frutos $(\mathrm{g})$ & 128,1 & 144,9 & ns & 22,1 \\
\hline Cor vermelha epiderme (\%) & 67,2 & 74,0 & $*$ & 8,7 \\
\hline Severidade de "russeting" $\left(\mathrm{cm}^{2}\right.$ fruto $\left.^{-1}\right)$ & 5,6 & 4,7 & ns & 21,9 \\
\hline Frutos com infecção de sarna (\%) & 64,6 & 17,7 & $* * *$ & 32,0 \\
\hline Frutos com queimadura por sol $(\%)$ & 3,5 & 0,8 & $*$ & 22,3 \\
\hline Danos por mosca-das-frutas (\%) & 0,0 & 69,4 & $* * *$ & 55,5 \\
\hline $\mathrm{N}^{\circ}$ de sementes/fruto & 6,8 & 6,7 & ns & 7,9 \\
\hline Índice de iodo-amido (1-5) & 3,3 & 3,0 & ns & 12,0 \\
\hline Firmeza de polpa $(\mathrm{N})$ & 78,4 & 81,4 & $*$ & 3,0 \\
\hline Sólidos solúveis ( ${ }^{\circ}$ Brix) & 11,7 & 10,8 & $*$ & 9,3 \\
\hline \multirow[t]{2}{*}{ Acidez titulável (\% de ácido málico) } & 0,38 & 0,42 & ns & 14,4 \\
\hline & \multicolumn{4}{|c|}{ 'Fuji' } \\
\hline Massa seca de 100 folhas (g) & 5,4 & 5,0 & ns & 10,3 \\
\hline Teor de clorofila (leitura SPAD) & 46,9 & 45,7 & $*$ & 2,2 \\
\hline $\mathrm{N}^{o}$ de cachos florais $\mathrm{PCSR}^{(2)}$ & 7,6 & 7,9 & ns & 36,8 \\
\hline $\mathrm{N}^{\circ}$ de frutos PCSR & 1,8 & 1,1 & $* *$ & 34,5 \\
\hline $\mathrm{N}^{o}$ de frutos $\mathrm{PCF}^{(3)}$ & 0,5 & 0,7 & ns & 45,8 \\
\hline Massa de frutos PCSR $(\mathrm{kg})$ & 0,26 & 0,14 & $* *$ & 38,8 \\
\hline Massa média de frutos (g) & 156,8 & 132,9 & $* * *$ & 8,5 \\
\hline Cor vermelha epiderme (\%) & 50,4 & 65,1 & $* * *$ & 6,4 \\
\hline Severidade de "russeting" $\left(\mathrm{cm}^{2}\right.$ fruto $\left.^{-1}\right)$ & 7,4 & 6,4 & ns & 24,6 \\
\hline Frutos com infecção de sarna (\%) & 41,0 & 6,0 & $* * *$ & 30,1 \\
\hline Frutos com queimadura por sol $(\%)$ & 1,0 & 1,0 & ns & 15,4 \\
\hline Danos por mosca-das-frutas $(\%)$ & 0,0 & 75,0 & $* * *$ & 51,6 \\
\hline $\mathrm{N}^{o}$ de sementes/fruto & 5,4 & 5,2 & ns & 11,5 \\
\hline Índice de iodo-amido (1-5) & 3,4 & 3,3 & ns & 5,8 \\
\hline Firmeza de polpa $(\mathrm{N})$ & 84,3 & 88,3 & $*$ & 3,4 \\
\hline Sólidos solúveis ( $\left.{ }^{\circ} \mathrm{Brix}\right)$ & 13,0 & 11,9 & $* *$ & 6,4 \\
\hline Acidez titulável (\% de ácido málico) & 0,43 & 0,44 & ns & 10,7 \\
\hline
\end{tabular}




\section{CONCLUSÕES}

O sistema orgânico de produção propiciou menor incidência de sarna em relação ao sistema convencional, nas cultivares Royal Gala e Fuji, nas condições do presente estudo, além de retardar o amadurecimento dos frutos e proporcionar frutos com maior percentual de cor vermelha na casca. Contudo, a alta incidência de danos por mosca-das-frutas ainda compromete o potencial da produção e a qualidade de maçãs orgânicas produzidas nas condições da Serra Catarinense. As aplicações de fungicidas cúpricos nos pomares orgânicos promovem acúmulo de $\mathrm{Cu}$ acima dos teores considerados normais nas folhas, mas não na casca e na polpa dos frutos. Maçãs orgânicas apresentaram maior suscetibilidade à ocorrência de distúrbios fisiológicos, uma vez que os frutos apresentaram menores teores de $\mathrm{Ca}$ e maiores valores das relações $\mathrm{K} / \mathrm{Ca}, \mathrm{Mg} / \mathrm{Ca}$ e $(\mathrm{K}+\mathrm{Mg}) / \mathrm{Ca}$ na polpa dos frutos.

\section{REFERENCIAS}

AMARANTE, C.V.T.; ARGENTA, L.C.; BASSO, C.; SUZUKI, A. Composição mineral de maçãs 'Gala' e 'Fuji' produzidas no Sul do Brasil. Pesquisa Agropecuária Brasileira, Brasília, v.47, n.47, p.550560, 2012.

AMARANTE, C.V.T.; CHAVES, D.V.; ERNANI, P.R. Análise multivariada de atributos nutricionais associados ao "bitter pit” em maçãs 'Gala'. Pesquisa Agropecuária Brasileira, Brasília, v.41, n.5, p.841846, 2006a.

AMARANTE, C.V.T.; CHAVES, D.V.; ERNANI, P.R. Composição mineral e severidade de "bitter pit” em maçãs 'Catarina'. Revista Brasileira de Fruticultura, Jaboticabal, v.28, n.1, p.51-54, 2006 b.

AMARANTE, C.V.T.; STEFFENS, C.A.; MAFRA, A.L.; ALBUQUERQUE, J.A. Yield and fruit quality of apple from conventional and organic production systems. Pesquisa Agropecuária Brasileira, Brasília, v.43, n.3, p.333-340, 2008.

DILMAGHANI, M.R.; MALAKOUTI, M.J.; NEILSEN, G.H.; FALLAHI, E. Interactive effects of potassium and calcium on $\mathrm{K} / \mathrm{Ca}$ ratio and its consequences on apple fruit quality in calcareous soils of Iran. Journal of Plant Nutrition, Philadelphia, v.27, n.7, p.1149-1162, 2004.
GARCIA, F.R.M.; CAMPOS, J.V.; CORSEUIL, E. Flutuação populacional de Anastrepha fraterculus (Weidmann, 1830) (Diptera, Tephritidae) na região oeste de Santa Catarina, Brasil. Revista Brasileira de Entomologia, Curitiba, v.47, n.3, p.415-420, 2003.

HOLB, I.J.; DREMÁKB, P.; BITSKEY, K.; GONDAB, I. Yield response, pest damage and fruit quality parameters of scab-resistant and scabsusceptible apple cultivars in integrated and organic production systems. Scientia Horticulturae, Amsterdam, v.145, p.109-117, 2012.

JÖNSSON, A. Organic apple production in Sweden: cultivation and cultivars. 2007. 33p. Doctoral Thesis - Department of Crop Science, Swedish University of Agricultural Sciences. Balsgård, Sweden. 2007.

LAYNE, D.R.; JIANG, Z.; RUSSHING, J.W. The influence of reflective film and retain on red skin coloration and maturity of 'Gala' apples. HortTechnology, Alexandria, v.12, n.4, p.640-644, 2002.

MALUCHE-BARETTA, C.R.D.; AMARANTE, C.V.T. do; KLAUBERG FILHO, O. Multivariate analysis of soil attributes of apple orchards under conventional and organic production systems. Pesquisa Agropecuária Brasileira, Brasília, v.41, n. 10, p.1531-1539, 2006.

PALMER, J.W.; DAVIES, S.B.; SHAW, P.W.; WÜNSCHE, J.N. Growth and fruit quality of 'Braeburn' apple trees as influenced by fungicide programs suitable for organic production. New Zealand Journal of Crop and Horticultural Science, Wellington, v.31, n.4, p.169-177, 2003.

PECK, G.M.; ANDREWS, P.K.; REGANOLD, J.P.; FELLMAN, J.K. Apple orchard productivity and fruit quality under organic, conventional, and integrated management. HortScience, Alexandria, v.41, n.1, p.99-107, 2006.

PECK, G.M.; MERWIN, I.A.; THIES, J.E.; SCHINDELBECK, R.R.; BROWN, M.G. Soil properties change during the transition to integrated and organic apple production in a New York orchard. Applied Soil Ecology, Amsterdam, v.48, n.1, p.1830, 2011.

REGANOLD, J.P.; GLOVER, J.D.; ANDREWS, 
P.K.; HINMAN, H.R. Sustainability of three apple production systems. Nature, London, v.410, n.19, p.926-930, 2001.

ROUSSOS, P.A.; GASPARATOS, D. Apple tree growth and overall fruit quality under organic and conventional management. Scientia Horticulturae, Amsterdam, v.123, n.2, p.247-252, 2009.

SAS INSTITUTE. SAS User's guide: statistics. Sofware Version 9.0. Cary, 2002.

TEDESCO, M.J.; GIANELLO, C.; BISSANI, C.A.; BOHNEN, H.; VOLKWEISS, S.J. Análises de solo, plantas e outros materiais. 2.ed. Porto Alegre: UFRGS, 1995, 174p.
TEIXEIRA, R.; AMARANTE, C.V.T.; BOFF, M.I.C.; RIBEIRO, L.G. Controle de pragas e doenças, maturação e qualidade de maçãs 'Imperial Gala' submetidas ao ensacamento. Revista Brasileira de Fruticultura, Jaboticabal, v.33, n.2, p.394-401, 2011.

WEIBEL, F.; WIDMER, F.; HUSISTEIN, A. Comparison of production systems: integrated and organic apple production. Part III: inner quality - composition and sensory. Obst und Weinbau, Traktoren, v.140, n.7, p.10-13, 2004. 